BMJ Open Sport \& Exercise Medicine

\title{
Rehabilitation following meniscal repair: a systematic review
}

\section{Robert C Spang III, ${ }^{1}$ Michael C Nasr, ${ }^{2}$ Amin Mohamadi, ${ }^{2}$ Joseph P DeAngelis, ${ }^{1}$ Ara Nazarian, ${ }^{2,3}$ Arun J Ramappa ${ }^{1}$}

\begin{abstract}
To cite: Spang III RC, Nasr MC, Mohamadi A, et al. Rehabilitation following meniscal repair: a systematic review. BMJ Open Sport \& Exercise Medicine 2018;4:e000212. doi:10.1136/ bmjsem-2016-000212
\end{abstract}

JPD, AN and AJR contributed equally.

JPD, AN and AJR are joint senior authors.

Accepted 9 February 2018

\section{Check for updates}

${ }^{1}$ Carl J. Shapiro Department of Orthopaedic Surgery, Beth Israel Deaconess Medical Center, Harvard Medical School, Boston, Massachusetts, USA

${ }^{2}$ Center for Advanced Orthopaedic Studies, Carl J. Shapiro Department of Orthopaedic Surgery, Beth Israel Deaconess Medical Center, Harvard Medical School, Boston, Massachusetts, USA

${ }^{3}$ Department of Orthopaedic Surgery, Yerevan State Medical University, Yerevan, Armenia

Correspondence to Dr Arun J Ramappa; aramappa@bidmc.harvard.edu

\section{ABSTRACT}

Objective To review existing biomechanical and clinical evidence regarding postoperative weight-bearing and range of motion restrictions for patients following meniscal repair surgery.

Methods and data sources Following the

Preferred Reporting Items for Systematic Reviews and Meta-Analyses guideline, we searched MEDLINE using following search strategy: ((/("Weight-Bearing/ physiology"[Mesh]) OR "Range of Motion, Articular"[Mesh]) OR "Rehabilitation"[Mesh])) AND ("Menisci, Tibial" [Mesh]). Additional articles were derived from previous reviews. Eligible studies were published in English and reported a rehabilitation protocol following meniscal repair on human We summarised rehabilitation protocols and patients' outcome among original studies.

Results Seventeen clinical studies were included in this systematic review. There was wide variation in rehabilitation protocols among clinical studies. Biomechanical evidence from small cadaveric studies suggests that higher degrees of knee flexion and weightbearing may be safe following meniscal repair and may not compromise the repair. An accelerated protocol with immediate weight-bearing at tolerance and early motion to non-weight-bearing with immobilising up to 6 weeks postoperatively is reported. Accelerated rehabilitation protocols are not associated with higher failure rates following meniscal repair.

Conclusions There is a lack of consensus regarding the optimal postoperative protocol following meniscal repair. Small clinical studies support rehabilitation protocols that allow early motion. Additional studies are needed to better clarify the interplay between tear type, repair method and optimal rehabilitation protocol.

\section{INTRODUCTION}

The menisci reduce stress by increasing the contact area between the femur and tibia. They buffer against axial, rotational and shearing forces about the knee during motion. ${ }^{1}$ The loss of meniscal tissue localises tibiofemoral contact and leads to progressive arthrosis ${ }^{23}$ and functional decline in the long term. ${ }^{4}$ To prevent these degenerative changes, meniscal repair has become more common. ${ }^{5}$ Kim et at documented a $25 \%$ increase in medial and lateral meniscal repairs between 1996 and 2006. Even though the majority of surgeries

\section{What is already known?}

The menisci reduce stress by increasing the contact area between the femur and tibia.

- Meniscal repair is becoming a more appealing treatment for meniscal injuries.

- There is a wide variation between postoperative rehabilitation protocols following meniscal repair.

What are the new findings?

- There is no consensus regarding postoperative rehabilitation protocol for meniscal repair.

- The quality of existing evidence is low.

- An accelerated rehabilitation protocol may be safely implemented for appropriate patients.

- Further studies are needed to determine an optimal rehabilitation protocol.

on the meniscus remain meniscectomies, Abrams et $a l^{7}$ found that between 2005 and 2011 more isolated meniscal repairs were performed in the USA without an increase in the number of meniscectomies. Meniscal repairs may be performed more frequently because there has been a significant advance in surgical techniques and repair devices (figure 1A-E). Historically, the gold standard for meniscal repair has been the inside-out technique (figure 1C,D). Long flexible needles are used to pass sutures through the tissue under arthroscopic guidance. ${ }^{8}$ The sutures are then retrieved using a separate incision and are tied over the joint capsule. This technique may place neurovascular structures at risk and requires an additional incision. ${ }^{9}$ To avoid the morbidity associated with an inside-out repair, 'all-inside' arthroscopic techniques have been developed (figure 1A,B). These include anchor-based repairs and suture-based repairs. The most popular of these designs employ pre-tied sutures between non-absorbable anchors. The anchors are deployed when an introducer is passed through the meniscal tear and the joint capsule (figure 1E,F). In a study of porcine meniscal repairs ${ }^{10}$ the inside-out 

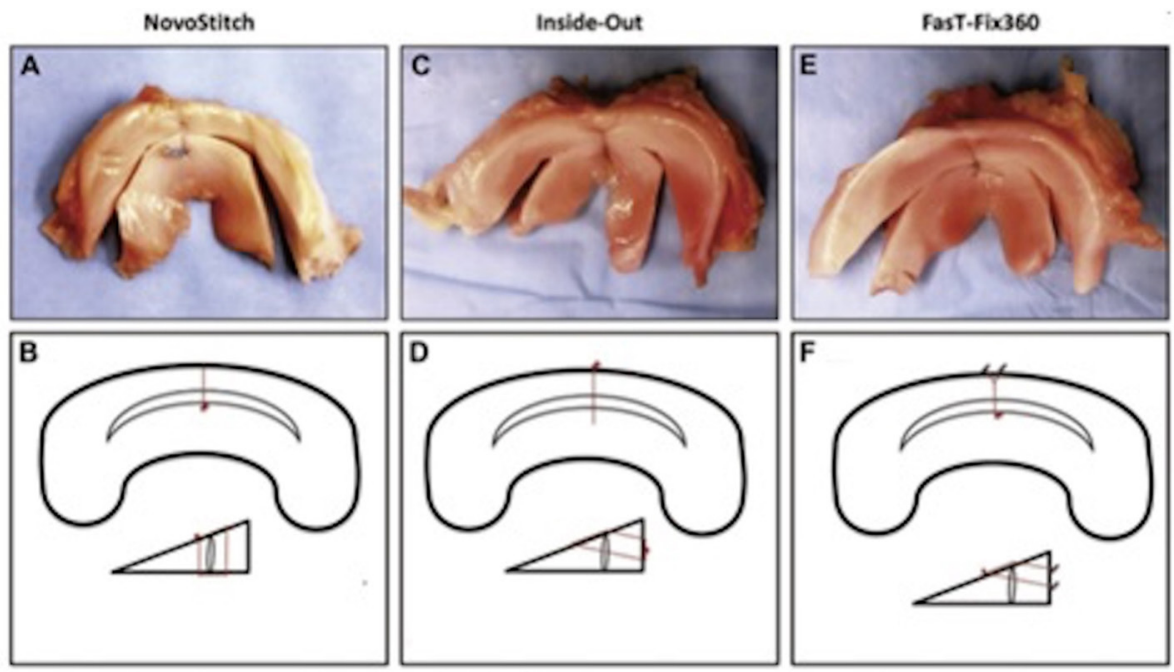

Figure 1 (A,B) All-inside suture-based repair, (C,D) inside-out suture repair and (E, F) anchor-based repair techniques.

technique was significantly stronger compared with recent all-inside repair devices. A study on fresh-frozen human menisci ${ }^{11}$ showed no difference. Although potentially less invasive than the inside-out technique, all-inside repairs can result in neurovascular injury, irritation from anchors and implant failure. ${ }^{12}$

Several factors may influence meniscal healing. The most important may be the meniscal blood supply. Scapinelli ${ }^{13}$ in 1968 and Arnoczky and Warren ${ }^{14}$ in 1982 described the limited peripheral blood supply to the outer one-third to one-quarter of the meniscus. From this finding, peripheral meniscal tears (ie, tears in the
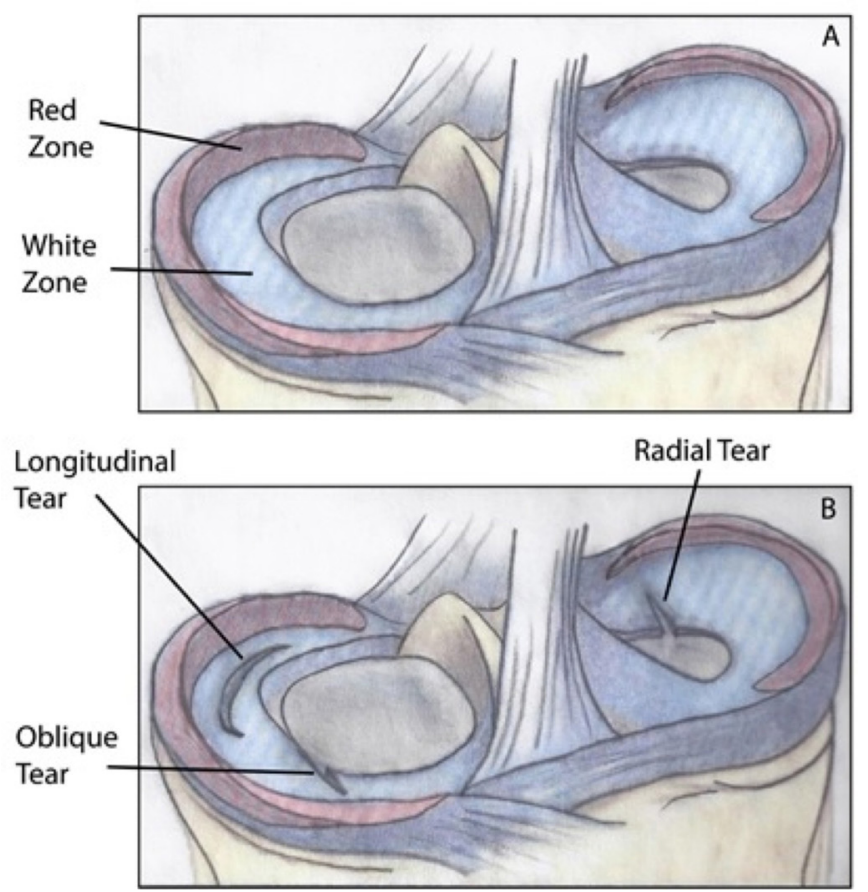

Figure 2 (A) Outer red zones receive blood supply; (B) longitudinal tears have a higher likelihood of being vascularized. 'red-red' zone) are felt to have better healing potential (figure 2A).

The timing and type of meniscal tear may also impact healing. Acute, traumatic tears tend to have higher healing rates than chronic, atraumatic tears. ${ }^{15}$ Longitudinal tears are more amenable to repair due to their vertical orientation (figure 2B), whereas radial tears extending to the central relatively avascular 'white-white' zone are more challenging. Some surgeons have reported success when repairing bucket-handle tears extending to the white-white zone. ${ }^{16}$

Age is another topic for consideration. Preserving meniscal tissue is particularly important for the longterm health of young athletes, and younger patients may have a higher healing potential. In one study of 26 patients aged 17 years or younger, none required a repeat surgery at an average of 5 years of follow-up. ${ }^{17} \mathrm{In}$ another report on two very young cases, meniscal repair for traumatic tears followed by limited weight-bearing rehabilitation resulted in a positive outcome. ${ }^{18}$ When age is not a factor, a meta-analysis investigating outcomes at least 5 years after meniscal repair showed a pooled failure rate of $23.1 \% .^{19}$

As biomechanical factors, postoperative range of motion (ROM) and weight-bearing status can impact meniscal healing after repair. The interplay between tear type and knee biomechanics can help define the most appropriate postoperative plan.

Restricting a patient's postoperative ROM intends to limit the risk of re-tear. Cadaveric studies have shown that femorotibial contact pressures increase with knee flexion..$^{20}$ If the ROM is restricted, the meniscal repair may be protected from increased mechanical stress. ${ }^{21}$ However, Richards et $a l^{22}$ investigated the effects of compressive loads in porcine longitudinal lateral meniscus repairs and found that weight-bearing reduced the meniscus and stabilised the repair. The highest compressive force occurred at full extension and the 


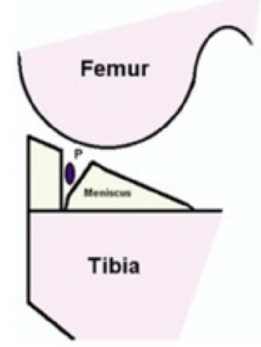

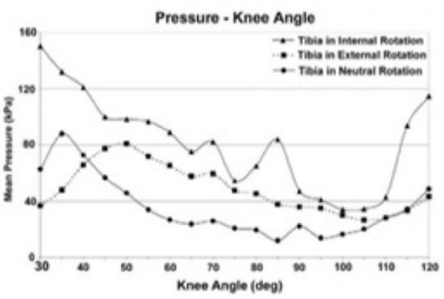

Figure 3 (A) The pressure transducer ' $P$ ' was placed in the lateral meniscal cut and the knee was cycled into flexion and extension. (B) Intrameniscal pressures were reflected in neutral, internal, and external rotation.

lowest was at $90^{\circ}$ of flexion. Beyond $100^{\circ}$ of flexion, it increased steadily (figure 3). Higher pressures were seen with internal rotation of the tibia, suggesting torsional forces may be different than axial loads. Conversely, for radial tears, axial loading might displace, rather than reduce, the injury. ${ }^{22}$ Of note, cadaveric investigations are limited in their ability to recreate the biomechanics of the knee in vivo and are not able to predict how specific rehabilitation protocols impact a meniscal repair. For this reason, rehabilitation following a meniscal repair is particularly conservative in an effort to protect the repaired meniscus.

Postoperative rehabilitation aims to foster healing after meniscal repair and facilitate the patient's return to full function. Generally, these programmes are initially focused on protecting the repair while regaining ROM and gradually introducing progressive strengthening en

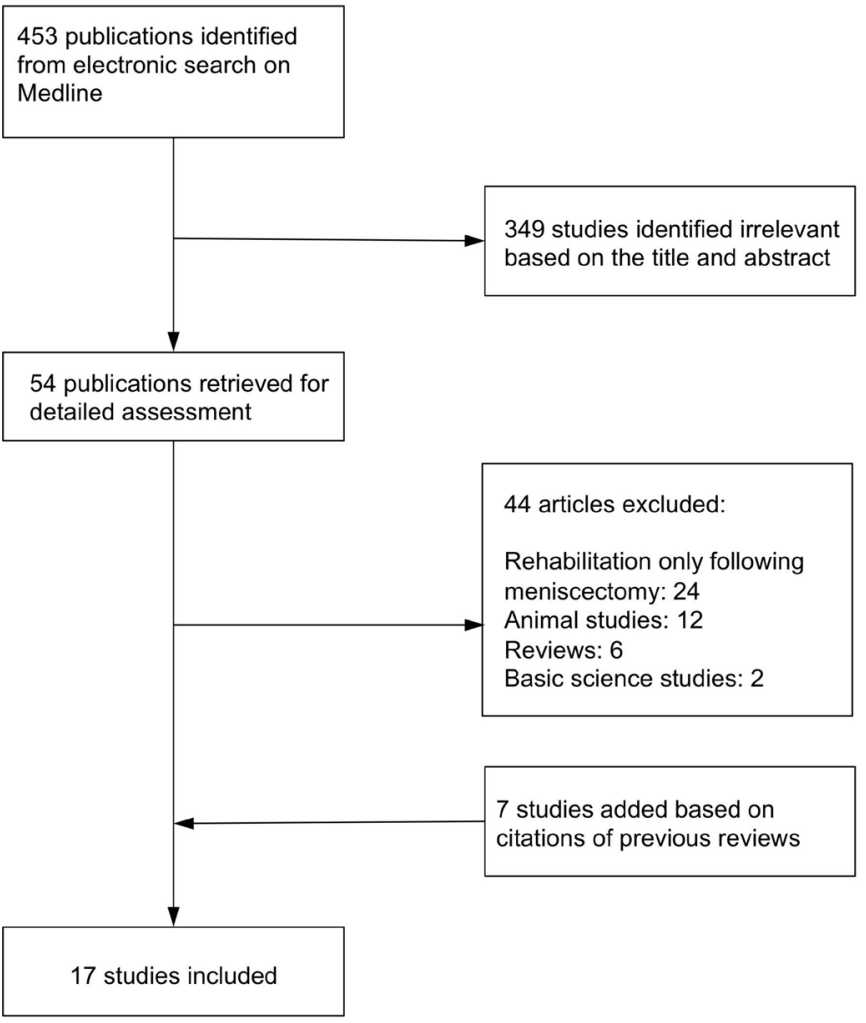

Figure 4 Flow chart of the systematic review. route to a return to preinjury activity level. At present, there is a paucity of evidence to support one best practice and there is a high degree of variability among postoperative rehabilitation programmes. Considering the increased frequency and evolution of meniscal repairs, this review intends to summarise the best-available evidence and practices regarding the postoperative care and rehabilitation of patients undergoing a meniscal repair.

\section{METHODS}

\section{Search strategy and data sources}

Following the Preferred Reporting Items for Systematic Reviews and Meta-Analyses guideline, on 15 June 2017 we conducted an electronic search on MEDLINE with the following search strategy: ( ( ("Weight-Bearing/physiology"[Mesh]) OR "Range of Motion, Articular"[Mesh]) OR "Rehabilitation" [Mesh])) AND ("Menisci, Tibial"[Mesh]). Additional sources included references of previous reviews. ${ }^{192324}$

A total of 453 studies were screened for eligibility. Only studies published in the English language in peer-reviewed journals were considered. Review papers, commentaries and studies on rehabilitation protocol following meniscectomy were excluded. Finally, 17 studies were included in the review (figure 4 ).

Bibliographic data, patients' characteristics, rehabilitation protocol and clinical outcome were recorded. Patient's outcome included meniscal healing, return to activities and clinical assessments based on the original studies. We did not confirm collected data by authors. The level of evidence for original studies is reported for each study. ${ }^{25}$

Cochrane tool for evaluating risk of bias was used for assessing the methodological quality of the included studies.

\section{RESULTS}

Seventeen clinical studies including 798 patients were reviewed in this systematic review. There was wide variation in methodological quality of clinical studies. The majority of studies had considerable risk of bias (table 1).

A restricted rehabilitation protocol was used for 438 patients. An accelerated protocol with immediate weightbearing at tolerance was used in 360 patients. Three studies compared restricted and accelerated protocol, which did not show any significant difference in complication rate or functional assessment (table 2).

Although a meta-analysis was not possible, it seems accelerated rehabilitation protocols are not associated with higher failure rates following meniscal repair.

\section{DISCUSSION}

Many rehabilitation programmes propose avoiding weight-bearing forces as an important goal in the immediate postoperative period to protect the repair from high compressive and shear forces. An MRI study of 
Table 1 Assessment of risk of bias using Cochrane tool

\begin{tabular}{|c|c|c|c|c|c|c|}
\hline & $\begin{array}{l}\text { Random sequence } \\
\text { generation } \\
\text { (selection bias) }\end{array}$ & $\begin{array}{l}\text { Allocation } \\
\text { concealment } \\
\text { (selection bias) }\end{array}$ & $\begin{array}{l}\text { Blinding of } \\
\text { participants and } \\
\text { personnel } \\
\text { (performance } \\
\text { bias) }\end{array}$ & $\begin{array}{l}\text { Blinding of } \\
\text { outcome } \\
\text { assessment } \\
\text { (detection bias) }\end{array}$ & $\begin{array}{l}\text { Incomplete } \\
\text { outcome data } \\
\text { (attrition bias) }\end{array}$ & $\begin{array}{l}\text { Selective } \\
\text { reporting } \\
\text { (reporting bias) }\end{array}$ \\
\hline Morgan and Casscells ${ }^{54}$ & - & - & - & - & + & + \\
\hline Morgan et $a l^{31}$ & - & - & - & - & + & + \\
\hline Barber $^{38}$ & - & - & - & - & + & + \\
\hline Fritz et $a l^{29}$ & - & - & - & - & + & + \\
\hline Mariani et $a l^{40}$ & - & - & - & - & + & + \\
\hline Shelbourne et $\mathrm{a} /{ }^{41}$ & - & - & - & - & + & + \\
\hline Barber and Click ${ }^{50}$ & - & - & - & - & $?$ & + \\
\hline O'Shea and Shelbourne ${ }^{47}$ & - & - & - & - & $?$ & + \\
\hline Kocabey et al $^{42}$ & - & - & - & - & + & + \\
\hline Bryant et al ${ }^{48}$ & $\begin{array}{c}+ \text { (Randomisation } \\
\text { for method of } \\
\text { repair) }\end{array}$ & + & $?$ & $?$ & $?$ & + \\
\hline Haklar et al ${ }^{45}$ & - & - & - & - & + & + \\
\hline Logan et $a l^{49}$ & - & - & - & - & + & + \\
\hline Lind et $a l^{35}$ & + & + & - & - & $?$ & + \\
\hline
\end{tabular}

-, high risk of bias; +, low risk of bias; ?, unclear risk of bias.

${ }^{*}$ Athletes under 17 years old.

†Very young children.

weight-bearing and non-weight-bearing knees found that the relative tibiofemoral movements of the loaded knee were similar to those in the unloaded knee. However, the medial femoral condyle moves approximately $4 \mathrm{~mm}$ anterior when the knee bends from full extension to $10^{\circ}$ of flexion while bearing weight. In the unloaded knee, the position of the medial femoral condyle did not change from extension to flexion. Laterally, the femoral condyle rolls forward $13 \mathrm{~mm}$ from $110^{\circ}$ to $60^{\circ}$ of flexion and 1 $\mathrm{mm}$ from $60^{\circ}$ to $0^{\circ}$ in the unloaded knee. ${ }^{26}$ In isolation, this pattern of motion suggests that non-weight-bearing knee flexion would be safe to $110^{\circ}$ for medial meniscal repairs and to $60^{\circ}$ for lateral meniscal repairs. However, this finding has not been validated clinically.

Becker $e t a l^{20}$ investigated changes in the meniscofemoral contact pressure after meniscal repair. Knees were loaded to approximately $50 \%$ of body weight, and meniscofemoral contact pressure was measured (Tekscan, Boston, Massachusetts, USA) while cycling the knee from extension to $90^{\circ}$ of flexion. They found that meniscofemoral pressures increased in both compartments as the knee flexed and that meniscal repair had no impact. Their study did not investigate the effect of this pressure difference on the meniscal repair, nor did they include torsional forces or higher impact loading. Ganley et $a l^{27}$ sought to further investigate knee flexion and loading on meniscal healing in a cadaveric model. They produced full-thickness posteromedial meniscal tears in cadaveric knees and imbedded metal markers into the tear following repair. Using CT scans, the marker position was assessed at $30^{\circ}, 60^{\circ}$ and $90^{\circ}$ of knee flexion after loading of $100 \mathrm{lbs}$ to simulate partial weight-bearing. They determined that neither flexion angle, loading nor suture had a significant impact (figures 5 and 6). In this way, accelerated rehabilitation programme with partial weight-bearing may be appropriate. Torsional forces, higher degrees of flexion and loads larger than $100 \mathrm{lbs}$ were not assessed.

Lin $e t a l^{28}$ sought to assess the effect of postoperative ROM following meniscal repair using a cadaveric model. They created a $2.5 \mathrm{~cm}$ posteromedial meniscal tear and repaired it with inside-out vertical mattress sutures (figure 7A). They measured the displacement at high degrees of flexion $\left(90^{\circ}, 110^{\circ}\right.$ and $\left.135^{\circ}\right)$ when loaded (figure 7B). Specimens were subjected to simulated open-chain flexion and extension with a load of $29 \mathrm{~N}$ applied to the hamstrings and $150 \mathrm{~N}$ to the quadriceps, exceeding the normal joint reactive force encountered 


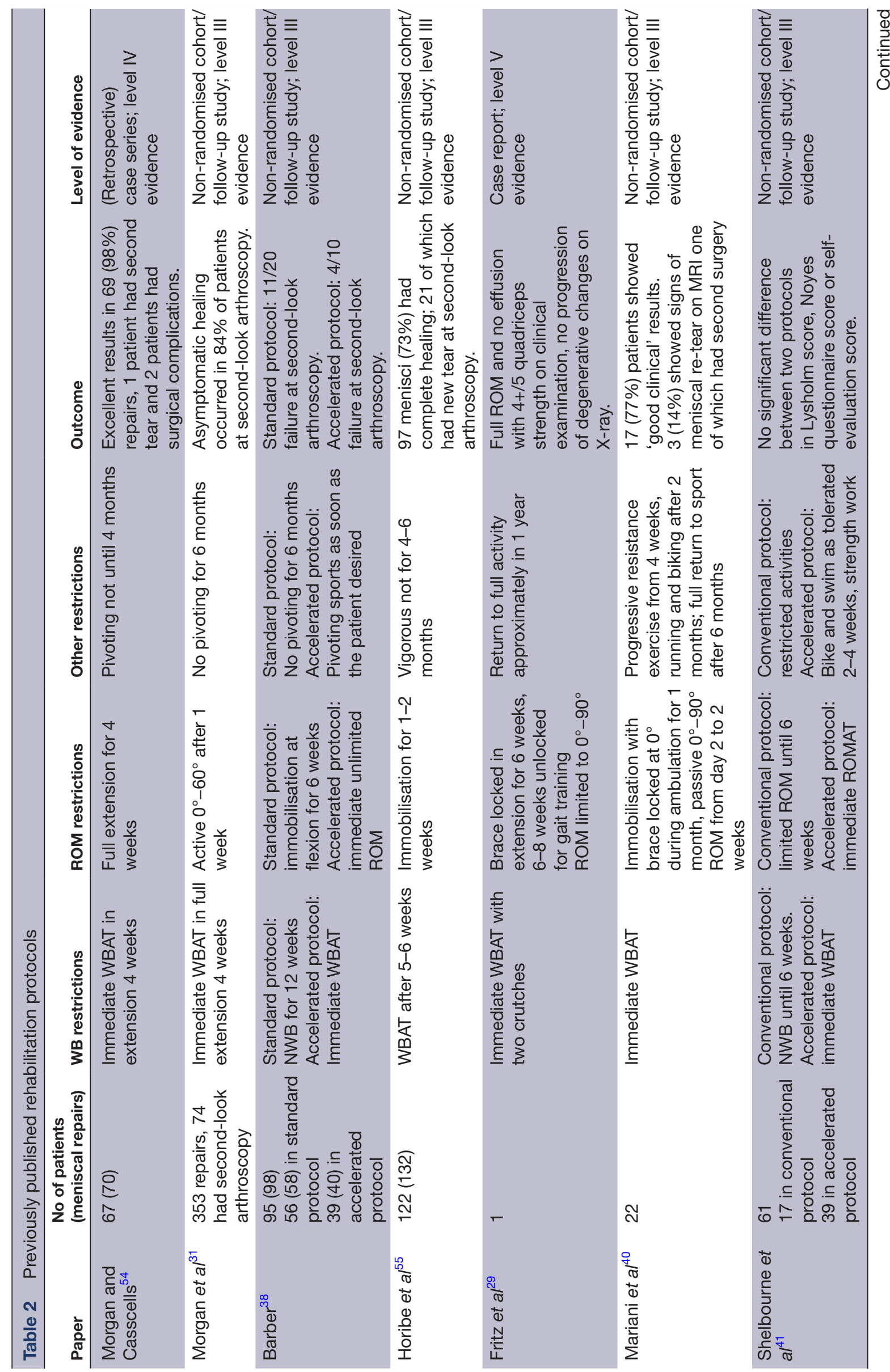




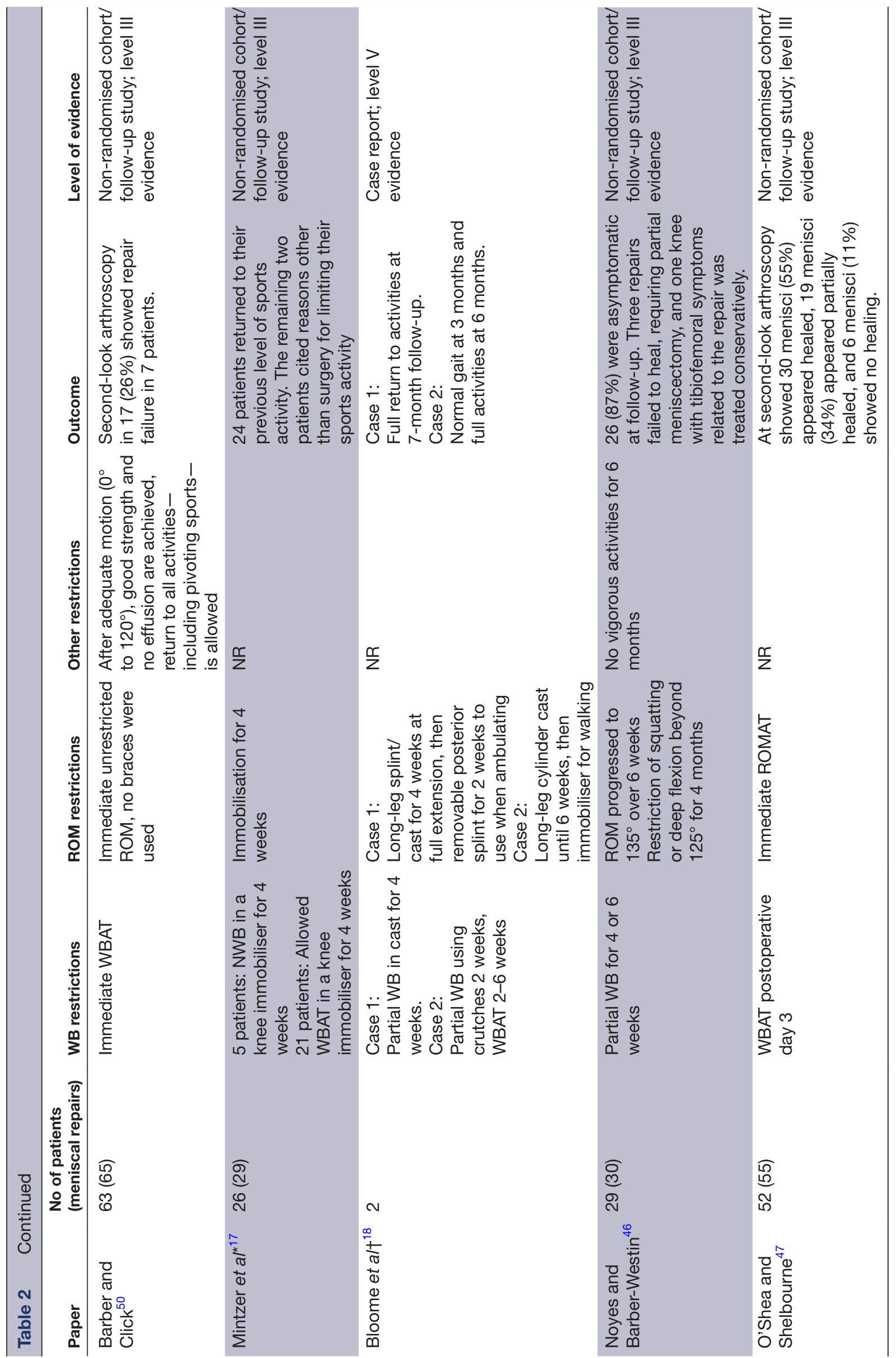




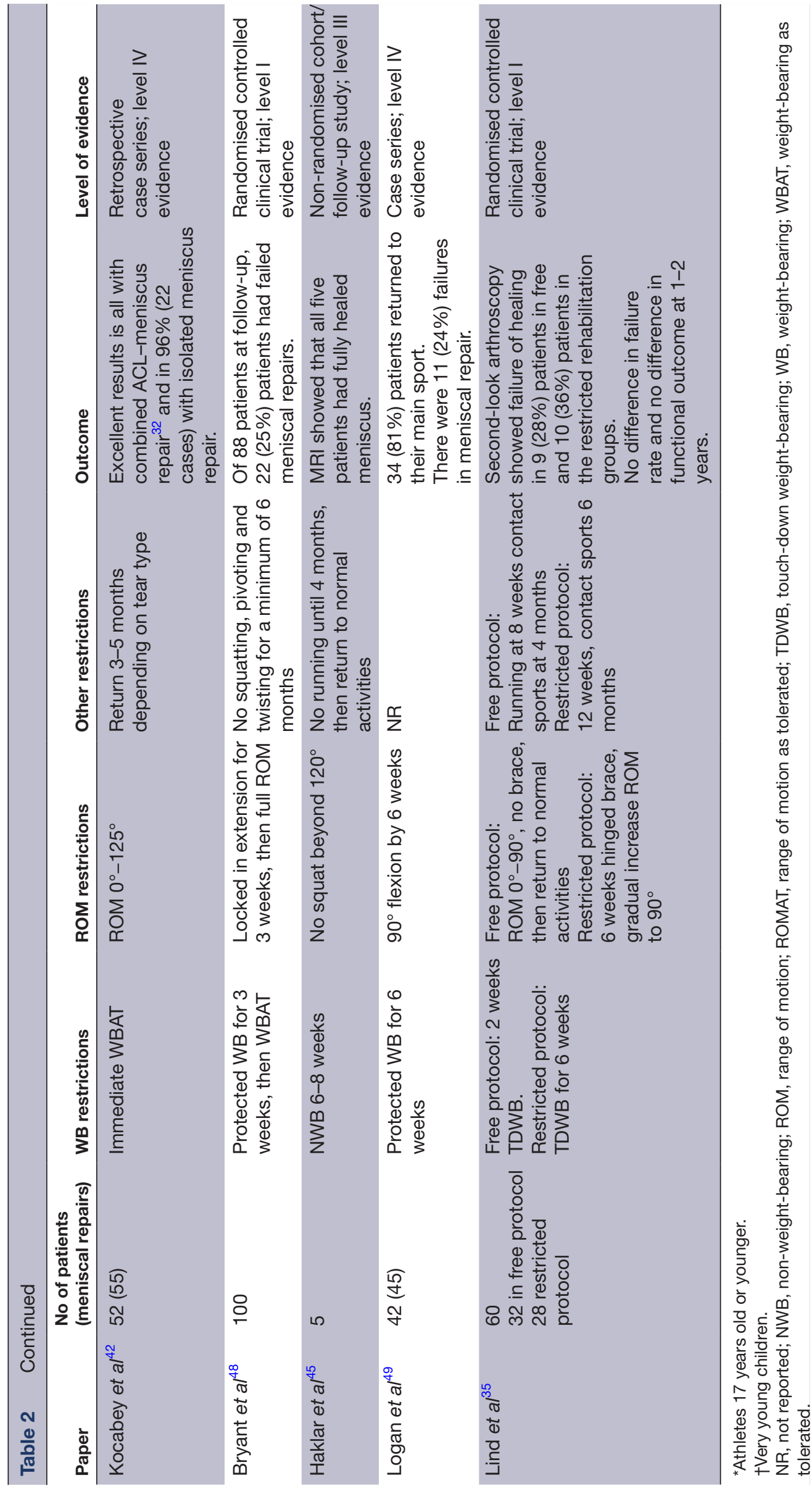




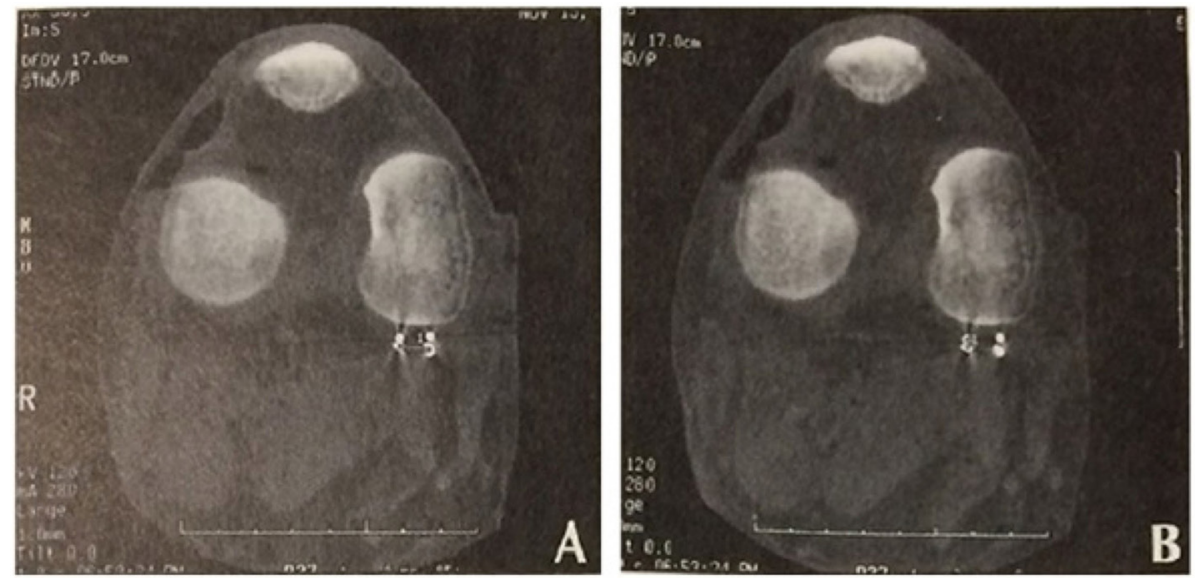

Figure 5 Transverse section of a cadaver knee under $100 \mathrm{lbs}$ of load at $60^{\circ}$. (A) Longitudinal and (B) transverse measurements are depicted between the markers.

during active knee flexion. ${ }^{28}$ In accordance with the findings of Richards $e t a t^{22}$ and Ganley $e t a l^{27}$ they found that neither the meniscal tear nor the meniscal repair demonstrated significant gapping. Rather they compressed in the transverse plane when flexed from $90^{\circ}$ to $135^{\circ} \mathrm{s}$ while subjected to physiologic loads. They conclude that 'non-restrictive un-resisted open chain ROM protocols do not place undue stress on meniscal repairs'.

Early weight-bearing might enhance the mechanical environment promoting healing and allowing earlier functional recovery and return to sport. ${ }^{22}$ It has been shown that early weight-bearing as tolerated and limited ROM resulted in acceptable outcomes (ie, Lysholm score of 71.5) at 17 month follow-up. ${ }^{29}$ While Becker's cadaveric findings may alleviate concern over iatrogenic cartilage damage from implants, the hypothetical danger of increased meniscofemoral pressures with knee flexion after meniscal repair has not been shown to impact clinical outcomes. ${ }^{20}$

Some authors recommend immobilisation in full extension, reportedly due to the observation that peripheral posterior horn tears move away from the capsule in flexion and reduce in extension. ${ }^{180-32}$ This recommendation stems from direct viewing of peripheral posterior horn tears using a $70^{\circ}$ arthroscope and observing the reduction of these tears during passive knee extension. However, this observation and the subsequent practice of immobilising meniscal repairs in extension has not been shown to be beneficial clinically and may not be relevant to other tear types. Some investigators recommend immobilisation in various degrees of flexion, ${ }^{32-34}$ and others still advocate for limited early motion. Despite more aggressive protocols allowing for free ROM immediately postoperatively, $90^{\circ}$ of flexion appears to be a comfortable restriction for surgeons. ${ }^{35}$ Up to $85 \%$ of the load travels through the menisci with the knee in $90^{\circ}$ of flexion, while less $(50 \%)$ of the load passes through the meniscus in extension. ${ }^{36}$

Additionally, meniscal dynamics using MRI three-dimensional reconstructions show that during knee flexion, the posterior excursion of the medial meniscus is $5.1 \mathrm{~mm}$ and the lateral meniscus is $11.2 \mathrm{~mm} .{ }^{37}$ However, a more recent study by Lin suggests that higher degrees of flexion may be safe. ${ }^{28}$ There is no clinical evidence that limiting weight-bearing and/or knee flexion improves healing rates. Long-term outcome studies are lacking.
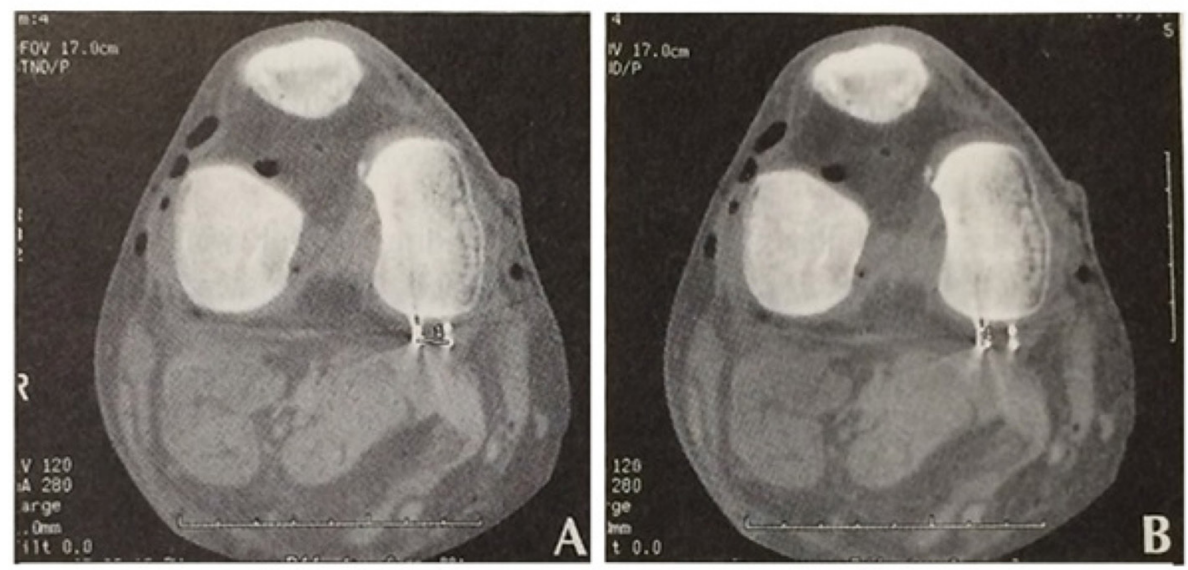

Figure 6 Transverse section of a cadaver knee without load at $60^{\circ}$. (A) Longitudinal and (B) transverse) measurements are depicted between the markers. 
A
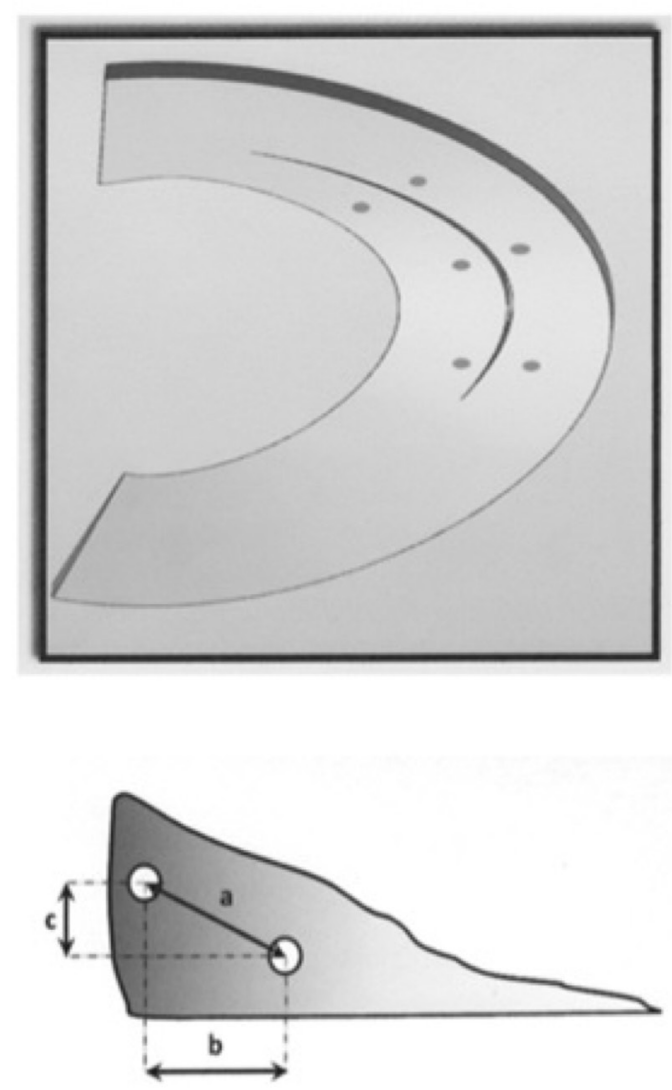

B

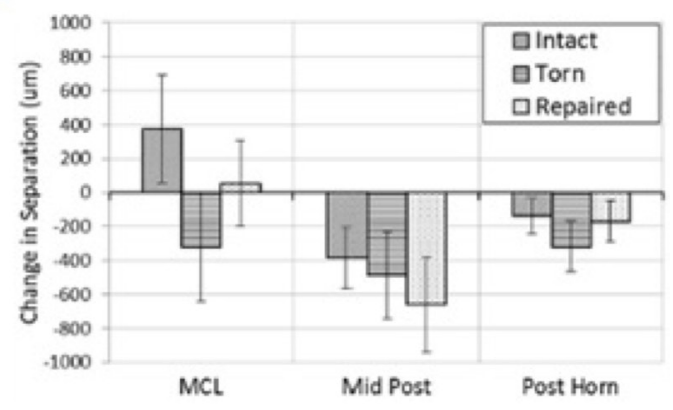

90-135 Transverse Plane

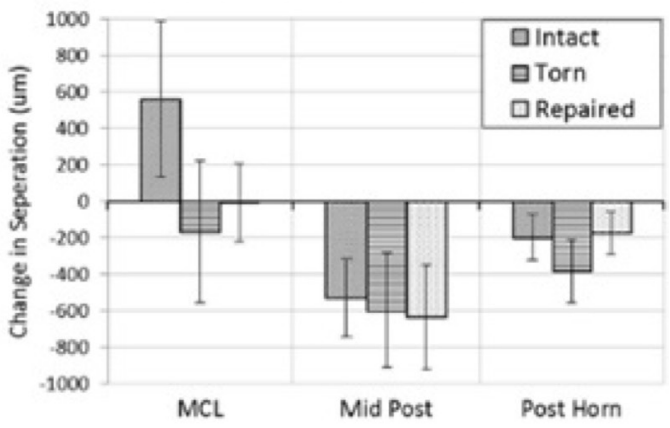

90-135 Vertical Change

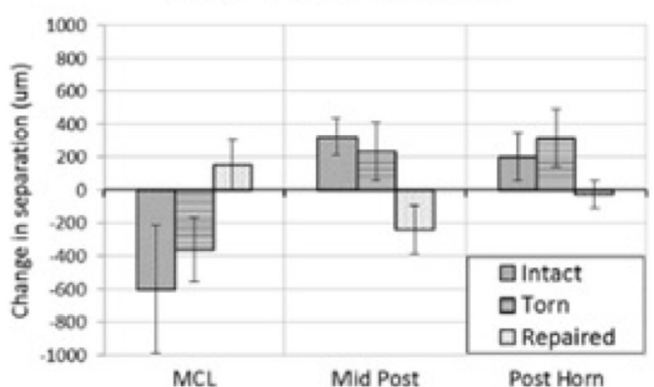

Figure 7 (A) Schematic of roentgen stereophotogrammetric analysis bead pair placement in relation to tear. Distances measured by vectors: $a-$ absolute, $b$-transverse and $c-$ vertical. (B) Changes in separation for each vector. Positive values indicate widening. Negative values indicate compression. MCL, medial collateral ligament region of posterior horn of medial meniscus; mid post, middle of posterior horn; post root, posterior root area of medial meniscus.

\section{Accelerated rehabilitation protocols}

A number of investigators have advocated for accelerated rehabilitation protocols (figure 8).$^{38-41}$ In a prospective randomised trial, Lind et $a l^{28}$ compared the impact of a 'free rehabilitation' regimen versus 'restricted rehabilitation'. 35 Sixty patients underwent isolated repair of a vertical meniscal lesion using an all-inside technique. They were randomised by rehabilitation regimens. The 'free' group was allowed to range the knee $0^{\circ}-90^{\circ}$ immediately while maintaining the knee in touch-down weight-bearing for 2 weeks, and weight-bearing as tolerated thereafter. They were allowed to return to contact sports at 4 months. The 'restricted' group wore a hinged brace for 6 weeks and gradually increased their ROM to $90^{\circ}$. They were touch-down weight-bearing for 6 weeks, followed by eventual return to sport at 6 months. The authors found no difference in the healing rate. At second-look arthroscopy, there were 9 and 10 failures in

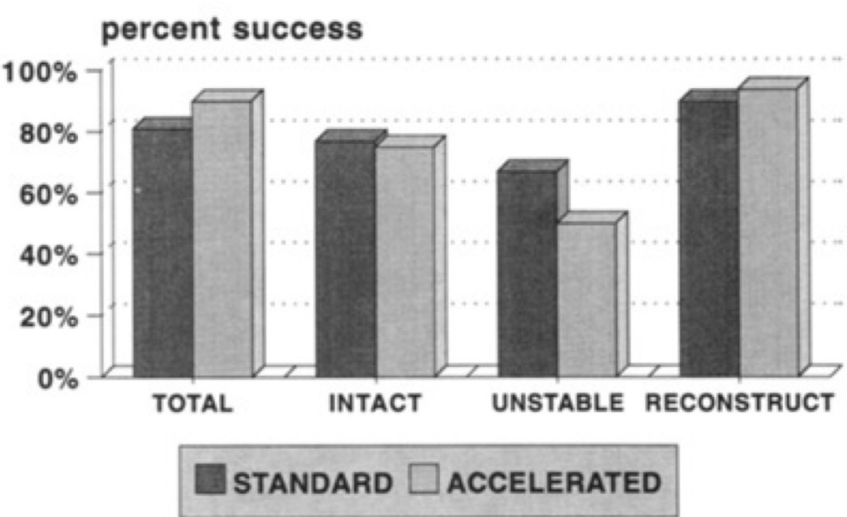

Figure 8 Meniscus repair success: standard versus accelerated. No difference in success rates exists between the standard accelerated rehabilitation groups. 
the free and restricted rehabilitation groups, respectively. There was no difference in functional outcome scores at 2 years. From this experience, the authors concluded that free rehabilitation was safe without a higher failure rate.

Kocabey et $a t^{42}$ reported excellent results using rehabilitation guidelines specific to the tear's characteristics. For anterior-posterior longitudinal tears less than $3 \mathrm{~cm}$, they promoted weight-bearing as tolerated without a brace. ROM progressed to $125^{\circ}$ between 3 and 6 weeks. For tears greater than $3 \mathrm{~cm}$, weight-bearing was allowed in a locked brace. ROM was limited to $0^{\circ}-125^{\circ}$ until 6 or 8 weeks. Return to sport was allowed after 3 months. For complex and radial tears, patients were required to wear a brace in which they were weight-bearing as tolerated ranging from $0^{\circ}$ to $125^{\circ}$ for 6 to 8 weeks. They returned to sport between 4 and 5 months.

Mariani et $a t^{40}$ followed 22 patients who underwent an outside-in meniscal repair. They were allowed to bear weight immediately without ROM restrictions. On re-examination with an MRI at an average of 28 months after surgery, only 3 of 22 patients showed signs of re-tear with greater than $1 \mathrm{~mm}$ of gapping. Based on this experience, they advocated for more aggressive rehabilitation regimens. ${ }^{36}$

There remains a concern regarding the safety of accelerated rehabilitation in the setting of a radial meniscal tear. Most studies investigating rehabilitation after meniscal repair have included patients with a longitudinal tear. However, since radial tears experience distraction forces and increased strain with axial loading, it is thought that a more conservative postoperative rehabilitation approach may be prudent in this setting. ${ }^{43}$ Choi et $a t^{44}$ and Haklar et $a t^{45}$ reported on their experiences repairing isolated radial tears of the lateral meniscus. Choi et $a l^{44}$ used a weight restriction protocol, whereas Haklar used a dual restriction protocol.

Overall, there is considerable variability in the rehabilitation following a meniscal repair. There is no clear consensus regarding the ideal programme (table 2). On the one hand, Noyes limited weight-bearing initially for 4-6 weeks, with ROM progressively advanced to $135^{\circ}$ of flexion over 6 weeks. ${ }^{46}$ At the other end of the spectrum, O'Shea and Shelbourne ${ }^{47}$ published favourable results after unrestricted ROM with weight-bearing as tolerated beginning 3 days after surgery. Similarly, Bryant et at8 allowed weight-bearing to tolerance with the knee locked in extension for 3 weeks, then WBAT with unlimited ROM thereafter. The impact of weight-bearing combined with twisting or pivoting movements on the repaired meniscus has not been adequately investigated. Furthermore, rehabilitation protocols with respect to meniscal repair with or without augmentation have not been evaluated.

\section{Return to sport}

The decision to repair a meniscus influences both the long-term health of the knee as well as the more immediate ability to return to activity. The postoperative treatment is an important consideration that should be discussed with the patient when considering a meniscus repair. Meniscal preservation offers long-term benefits. However, because the recovery requires a longer period of immobilisation with restrictions and delays the return to sport, some athletes might not want to have a meniscal repair. In one study of 45 meniscal repairs in elite athletes, $81 \%$ returned to sports, with the vast majority back to their prior sporting level. ${ }^{49}$ The mean return to sport was 5.6 months (range 3-8 months) for an isolated meniscal tear, compared with 11.8 months for ACL reconstruction with meniscal repair.

\section{Meniscal repair in association with ACL reconstruction}

Accelerated, or aggressive, rehabilitation is important following ACL reconstruction to improve ROM. ${ }^{41}$ Several studies have shown that accelerated rehabilitation is safe following ACL reconstruction with meniscal repair. ${ }^{40} 50$ In a series of 63 consecutive patients, 58 meniscal tears were repaired arthroscopically using an inside-out technique at the time of ACL reconstruction. Barber $e t a b^{5^{0}}$ promoted a rapid return to full function. Patients were aggressively rehabilitated to playing non-contact pivoting sports at 10-12 weeks, with unlimited activity using a derotational brace as early as 3-4 months once adequate motion $\left(0^{\circ}-120^{\circ}\right)$, good strength and no effusion were achieved. With regards to meniscal repairs, the authors noted a lack of consensus regarding rehabilitation protocols and called restrictions into question.

Many surgeons do not restrict patients after meniscal repair in the setting of concurrent ACL reconstructions. It has been hypothesised that meniscal repairs benefit from an abundance of healing factors due to the intra-articular bleeding present during an ACL reconstruction. In a matched cohort study by Wasserstein $e t a l^{51}$ the patient cohort with concomitant ACL reconstruction was found to have a meniscal reoperation rate of $9.7 \%$ compared with $16.7 \%$ in the meniscus repair alone cohort. Conversely, in an ACL-deficient knee, meniscal repairs are prone to failure due to the persistent mechanical stress on the tissue..$^{315253}$

\section{CONCLUSION}

Meniscal repair is an important procedure that aims to preserve tissue and prevent future arthrosis. While treatment may alleviate symptoms and allow for a timely return to activity, there is a lack of consensus regarding the optimal postoperative rehabilitation protocol. Moreover, there is scarce evidence supporting many current practices. Biomechanical evidence suggests that high degrees of knee flexion may be safe, but these data are limited to a few cadaveric studies. The impact of rotation and torsion forces has not been determined, but have implications for the return to sport and work. It is unclear whether larger joint forces associated with running or jumping threaten the meniscal repair.

An accelerated rehabilitation protocol may be safely implemented for appropriate patients, but it is unclear how the type of meniscal tear and the repair technique should affect the postoperative programme. Additional biomechanical studies are needed to better clarify the 
interplay between tear type, repair method, knee loading, knee positioning and torsional forces. Clinical studies investigating these specific elements will help to optimise patient outcomes.

Contributors RCS, MCN, AM, JPD, AN and AJR developed the idea of the study. RCS, MCN and AM collected the data. JPD, AN and AJR approved the accuracy of the collected data. RCS, MCN and AM prepared first draft of the manuscript. JPD, AN and AJR provided clinical and biomechanical insight to the study. All authors approved final manuscript.

Funding The authors have not declared a specific grant for this research from any funding agency in the public, commercial or not-for-profit sectors.

Competing interests None declared.

Patient consent Not required.

Provenance and peer review Not commissioned; externally peer reviewed.

Data sharing statement This is a systematic review of previously published studies. All studies are available to the public.

Open Access This is an Open Access article distributed in accordance with the Creative Commons Attribution Non Commercial (CC BY-NC 4.0) license, which permits others to distribute, remix, adapt, build upon this work non-commercially, and license their derivative works on different terms, provided the original work is properly cited and the use is non-commercial. See: http://creativecommons.org/ licenses/by-nc/4.0/

C Article author(s) (or their employer(s) unless otherwise stated in the text of the article) 2018. All rights reserved. No commercial use is permitted unless otherwise expressly granted.

\section{REFERENCES}

1. Kurosawa H, Fukubayashi T, Nakajima H. Load-bearing mode of the knee joint: physical behavior of the knee joint with or without menisci. Clin Orthop Relat Res 1980;149:283-90.

2. Fairbank TJ. Knee joint changes after meniscectomy. J Bone Joint Surg Br 1948;30B:664-70.

3. Seitz AM, Lubomierski A, Friemert B, et al. Effect of partial meniscectomy at the medial posterior horn on tibiofemoral contact mechanics and meniscal hoop strains in human knees. J Orthop Res 2012;30:934-42.

4. Roos EM, Ostenberg A, Roos H, et al. Long-term outcome of meniscectomy: symptoms, function, and performance tests in patients with or without radiographic osteoarthritis compared to matched controls. Osteoarthritis Cartilage 2001;9:316-24.

5. Stein T, Mehling AP, Welsch F, et al. Long-term outcome after arthroscopic meniscal repair versus arthroscopic partial meniscectomy for traumatic meniscal tears. Am J Sports Med 2010;38:1542-8.

6. Kim S, Bosque J, Meehan JP, et al. Increase in outpatient knee arthroscopy in the United States: a comparison of National Surveys of Ambulatory Surgery, 1996 and 2006. J Bone Joint Surg Am 2011;93:994-1000.

7. Abrams GD, Frank RM, Gupta AK, et al. Trends in meniscus repair and meniscectomy in the United States, 2005-2011. Am J Sports Med 2013;41:2333-9.

8. Johnson D, Weiss B. Meniscal repair using the inside-out suture technique. Sports Med Arthrosc Rev 2012;20:68-76.

9. Baena AE, Castilla BM, Fernandez JS, et al. Inside-out medial meniscus suture: an analysis of the risk of injury to the popliteal neurovascular bundle. Arthroscopy 2011;27:516-21.

10. Rosso C, Kovtun K, Dow W, et al. Comparison of all-inside meniscal repair devices with matched inside-out suture repair. Am J Sports Med 2011;39:2634-9.

11. Seitz A, Kasisari R, Claes L, et al. Forces acting on the anterior meniscotibial ligaments. Knee Surg Sports Traumatol Arthrosc 2012;20:1488-95.

12. Grant JA, Wilde J, Miller BS, et al. Comparison of inside-out and all-inside techniques for the repair of isolated meniscal tears: a systematic review. Am J Sports Med 2012;40:459-68.

13. Scapinelli $R$. Studies on the vasculature of the human knee joint. Acta Anat 1968;70:305-31.

14. Arnoczky SP, Warren RF. Microvasculature of the human meniscus. Am J Sports Med 1982;10:90-5.

15. Venkatachalam S, Godsiff SP, Harding ML. Review of the clinical results of arthroscopic meniscal repair. Knee 2001;8:129-33.
16. Gallacher PD, Gilbert RE, Kanes G, et al. White on white meniscal tears to fix or not to fix? Knee 2010;17:270-3.

17. Mintzer CM, Richmond JC, Taylor J. Meniscal repair in the young athlete. Am J Sports Med 1998;26:630-3.

18. Bloome DM, Blevins FT, Paletta GA, et al. Meniscal repair in very young children. Arthroscopy 2000;16:545-9.

19. Nepple JJ, Dunn WR, Wright RW. Meniscal repair outcomes at greater than five years: a systematic literature review and metaanalysis. J Bone Joint Surg Am 2012;94:2222-7.

20. Becker R, Wirz D, Wolf C, et al. Measurement of meniscofemoral contact pressure after repair of bucket-handle tears with biodegradable implants. Arch Orthop Trauma Surg 2005;125:254-60.

21. Anderson DR, Gershuni DH, Nakhostine M, et al. The effects of nonweight-bearing and limited motion on the tensile properties of the meniscus. Arthroscopy 1993;9:440-5.

22. Richards DP, Barber FA, Herbert MA. Compressive loads in longitudinal lateral meniscus tears: a biomechanical study in porcine knees. Arthroscopy 2005;21:1452-6.

23. VanderHave KL, Perkins C, Le M. Weightbearing versus nonweightbearing after meniscus repair. Sports Health 2015;7:399-402.

24. Cavanaugh JT, Killian SE. Rehabilitation following meniscal repair. Curr Rev Musculoskelet Med 2012;5:46-58.

25. Hurwitz SR, Slawson D, Shaughnessy A. Orthopaedic information mastery: applying evidence-based information tools to improve patient outcomes while saving orthopaedists' time. J Bone Joint Surg Am 2000;82:888-94.

26. Hill PF, Vedi V, Williams A, et al. Tibiofemoral movement 2: the loaded and unloaded living knee studied by MRI. J Bone Joint Surg Br 2000;82:1196-8.

27. Ganley T, Arnold C, McKernan D, et al. The impact of loading on deformation about posteromedial meniscal tears. Orthopedics 2000;23:597-601.

28. Lin DL, Ruh SS, Jones HL, et al. Does high knee flexion cause separation of meniscal repairs? Am J Sports Med 2013;41:2143-50.

29. Fritz JM, Irrgang JJ, Harner CD. Rehabilitation following allograft meniscal transplantation: a review of the literature and case study. $J$ Orthop Sports Phys Ther 1996;24:98-106.

30. Busenkell GL, Lee CS. Arthroscopic meniscal repair: a posterior cannulated technique. Arthroscopy 1992;8:247-53.

31. Morgan CD, Wojtys EM, Casscells CD, et al. Arthroscopic meniscal repair evaluated by second-look arthroscopy. Am J Sports Med 1991;19:632-8.

32. Sommerlath K, Hamberg P. Healed meniscal tears in unstable knees. A long-term followup of seven years. Am J Sports Med 1989;17:161-3.

33. Marzo J, Warren R, Arnoczky S, et al. Arthroscopic meniscal repair review of the outside-in technique. Am J Knee Surg 1991;4:164-71.

34. Scott GA, Jolly BL, Henning CE. Combined posterior incision and arthroscopic intra-articular repair of the meniscus. An examination of factors affecting healing. J Bone Joint Surg Am 1986;68:847-61.

35. Lind $M$, Nielsen $T$, Faunø $\mathrm{P}$, et al. Free rehabilitation is safe after isolated meniscus repair: a prospective randomized trial comparing free with restricted rehabilitation regimens. Am J Sports Med 2013;41:2753-8.

36. Ahmed AM. A pressure distribution transducer for in-vitro static measurements in synovial joints. J Biomech Eng 1983;105:309-14.

37. Thompson WO, Thaete FL, Fu FH, et al. Tibial meniscal dynamics using three-dimensional reconstruction of magnetic resonance images. Am J Sports Med 1991;19:210-6.

38. Barber FA. Accelerated rehabilitation for meniscus repairs. Arthroscopy 1994;10:206-10.

39. Kozlowski EJ, Barcia AM, Tokish JM. Meniscus repair: the role of accelerated rehabilitation in return to sport. Sports Med Arthrosc Rev 2012;20:121-6.

40. Mariani PP, Santori N, Adriani E, et al. Accelerated rehabilitation after arthroscopic meniscal repair: a clinical and magnetic resonance imaging evaluation. Arthroscopy 1996;12:680-6.

41. Shelbourne KD, Patel DV, Adsit WS, et al. Rehabilitation after meniscal repair. Clin Sports Med 1996;15:595-612.

42. Kocabey Y, Nyland J, Isbell WM, et al. Patient outcomes following T-Fix meniscal repair and a modifiable, progressive rehabilitation program, a retrospective study. Arch Orthop Trauma Surg 2004;124:592-6.

43. Jones RS, Keene GC, Learmonth DJ, et al. Direct measurement of hoop strains in the intact and torn human medial meniscus. Clin Biomech 1996;11:295-300.

44. Choi NH, Kim TH, Son KM, et al. Meniscal repair for radial tears of the midbody of the lateral meniscus. Am J Sports Med 2010;38:2472-6. 
45. Haklar U, Kocaoglu B, Nalbantoglu U, et al. Arthroscopic repair of radial lateral meniscus [corrected] tear by double horizontal sutures with inside-outside technique. Knee 2008;15:355-9.

46. Noyes FR, Barber-Westin SD. Arthroscopic repair of meniscus tears extending into the avascular zone with or without anterior cruciate ligament reconstruction in patients 40 years of age and older. Arthroscopy 2000;16:822-9.

47. O'Shea JJ, Shelbourne KD. Repair of locked bucket-handle meniscal tears in knees with chronic anterior cruciate ligament deficiency. Am J Sports Med 2003;31:216-20.

48. Bryant D, Dill J, Litchfield R, et al. Effectiveness of bioabsorbable arrows compared with inside-out suturing for vertical, reparable meniscal lesions: a randomized clinical trial. Am J Sports Med 2007;35:889-96.

49. Logan M, Watts M, Owen J, et al. Meniscal repair in the elite athlete: results of 45 repairs with a minimum 5-year follow-up. Am J Sports Med 2009;37:1131-4.
50. Barber FA, Click SD. Meniscus repair rehabilitation with concurrent anterior cruciate reconstruction. Arthroscopy 1997;13:433-7.

51. Wasserstein D, Dwyer T, Gandhi R, et al. A matched-cohort population study of reoperation after meniscal repair with and without concomitant anterior cruciate ligament reconstruction. Am J Sports Med 2013;41:349-55.

52. DeHaven KE, Black KP, Griffiths HJ. Open meniscus repair. Technique and two to nine year results. Am J Sports Med 1989;17:788-95.

53. Hamberg P, Gillquist J, Lysholm J. Suture of new and old peripheral meniscus tears. J Bone Joint Surg Am 1983;65:193-7.

54. Morgan CD, Casscells SW. Arthroscopic meniscus repair: a safe approach to the posterior horns. Arthroscopy 1986;2:3-12.

55. Horibe S, Shino K, Nakata K, et al. Second-look arthroscopy after meniscal repair. Review of 132 menisci repaired by an arthroscopic inside-out technique. J Bone Joint Surg Br 1995;77:245-9. 